\title{
LAS DIVISORIAS DE AGUAS COMO ELEMENTOS DEL PAISAJE
}

\author{
José Carlos GONZALEZ HIDALGO \\ Universidad de Zaragoza
}

\begin{abstract}
Resumen: Ante la escasa utilidad que en ocasiones presenta el análisis de la red de drenaje en los medios de parcas precipitaciones, se plantea el estudio de las divisorias como paliativo de esta situación, teniendo presente que en ellas es donde en definitiva se genera el flujo.
\end{abstract}

\begin{abstract}
Given that the drainage network analysis in some circumstances is not efficient to know the patterns of runoff (areas with low rainfall), in this work is presented a divide line study having in mind that the divide lines are the places from where the overland flow begin.
\end{abstract}

PALABRAS CLAVE: Drenaje en superficie. Divisorias. Semiárido. Violada.

Sumario: Introducción.- Planteamiento metodológico.- Area de estudio.- El relieve y la capacidad de movilización del flujo.- Conclusiones.- Bibliografía.

\section{INTRODUCCION}

El flujo de las aguas en superficie, bajo cualquiera de sus formas (arroyada laminar, concentrada, escorrentia permanente), es uno de los principales agentes del modelado terrestre.

Los factores que controlan su distribución en el espacio y en el tiempo, son de dos tipos. Por una parte el clima, principalmente mediante el aporte de precipitación, definiendo sobre todo las pautas temporales. Por otra parte, aquellos factores más relacionados con su distribución en el espacio: litología, cubierta vegetal y topografía (KNIGHTON, 1984).

El análisis de la influencia que tiene el relieve en los procesos morfogenéticos, (léase distribución de flujo superficial), lleva directamente a considerar dos aspectos: 
- En primer lugar hay que tener presente que sobre él se ejercen los propios procesos. Es quien más visiblemente, y en última instancia, se ve afectado, siendo su expresión más clara.

- En segundo lugar, el mismo participa en los procesos en virtud de sus caracteres. Adquiere así una posición relativa respecto a los demás elementos en la trama de relaciones que los constituyen.

Por consiguiente, el relieve, elemento estático y poco cambiante en la escala humana de observación, pasa a ocupar una dimensión dinámica, y deja de ser sujeto paciente para convertirse en un elemento activo dentro de la trama de relaciones de los procesos morfogenéticos.

Los principales atributos topográficos (pendiente y su desarrollo longitudinal) han sido desde muy temprano reconocidos como fundamentales en lo que respecta a la producción del flujo en superficie. Por consiguiente, lo han sido en la producción y arrastre de sedimentos, procesos ambos considerados como los más expresivos de entre los actuales en la creación de formas.

Baste recordar en este sentido los tempranos modelos de ZINGG (1940) o MUSGRAVE (1947) (ambos en THORNES 1976), las modificaciones efectuadas por SMITH y BRETHERTON (1972, en JONES 1987), BAND (1985), su aplicación reciente en trabajos en España (THORNES y GILMAN, 1983) o su inclusión como factor (denominado LS) en el modelo de predicción de sedimentos más utilizado: la USLE (WISCHMEIER and SMITH, 1960; vid también MORGAN 1979, p. 117).

Indirectamente, otros análisis topográficos han venido siendo utilizados en esta línea. La vía de análisis se ha centrado en lo que al inicio se apuntaba, es decir: entendiendo la topografía como un factor de distribución del flujo, v.g. en el estudio de las redes de drenaje, mediante las metodologías de HORTON (1945), STRAHLER (1964), SCHREVE (1966) y SCHEIDEGGER (1968) (todos ellos en MOPU 1984). De todas ellas sin duda la más empleada, pese a las críticas puestas de manifiesto por diversos autores (vid KNIGHTON, 1984), es la propuesta por STRAHLER.

En cualquier caso, el método en esencia supone un cambio de escala, y ha demostrado su utilidad en los estudios de grandes extensiones (GREGORY, 1976; 
MORGAN 1976, ambos en relación a variables climáticas); en la comparación de diferencias litológicas e hipsométricas (un ejemplo reciente dentro de la comunidad aragonesa se puede ver en GONZALEZ HIDALGO 1990); o en la comparación de diferencias relativas en la respuesta hidrológica entre cuencas (AVENA et alt. 1967), con interesantes aplicaciones en España (vid. JARDI, 1986).

Al cambio de escala espacial se suma, además, el cambio de escala temporal, dado que el establecimiento de la red de drenaje en superficie es un proceso de mucha más larga duración que cualquiera de los procesos actuales.

En definitiva, el análisis de la red de drenaje en su estado actual supone un acercamiento indirecto (efecto final, aunque su evolución no tiene porque estar paralizada) a las condiciones del establecimiento de su asentamiento. A la vez supone también un aporte indirecto al conocimiento de las condiciones de la respuesta hidrológica actual de un área. Y por consiguiente enlaza directamente con el estudio de los procesos morfogenéticos (una revisión bibliográfica de este tema se encuentra en MITCHELL y BUBENZER, 1980).

El problema surge cuando la red superficial es poco nítida, y, ni en el estudio cartográfico, con los inconvenientes de escala o tipo de Mapa (vid. GOUDIE et alt., 1981 pp. 48 y 49), ni con la fotointerpretación, ni en los reconocimientos directos en el terreno, se vislumbra la plasmación en el espacio de las líneas de flujo.

Este hecho es un problema dentro de las áreas semiáridas y áridas, donde la ausencia de escorrentra permanente y puede dar lugar en muchas ocasiones a la falta de cauces nítidos, o, cuando éstos se pueden observar, a la imposibilidad de precisar sus límites.

Por consiguiente, en tales áreas, la metodología del análisis topográfico en relación al estudio de la distribución del flujo superficial vía redes de drenaje, debe reorientarse. En este sentido apunta el presente trabajo, que no supone más que abordar el mismo problema, (el estudio de la distribución del flujo superficial como agente de erosión en relación a la topografía), desde una óptica distinta. 


\section{PLANTEAMIENTO METODOLOGICO.}

Es sobradamente conocido que en los ambientes de escasas precipitaciones (áridos y semiáridos $<500 \mathrm{~mm}$ año) el flujo en superficie es uno de los principales agentes de erosión. Pero a su vez se trata de flujos temporales con una dependencia directa de los ritmos pluviales. En estas áreas, junto a cauces de incisión lineal producto de flujos concentrados, las manifestaciones de otros tipos de flujos superficiales (laminar, en regueros, etc.) son mucho más evidentes.

En cuanto fenómeno temporal y espasmódico, ante la ausencia de cursos permanentes y ante el dominio de flujos de caracteres incipientes, parece más apropiado el análisis de la relación topográfica y procesos morfogenéticos (vía arroyada superficial) a partir del estudio desde donde se produce el flujo, más que el estudio del donde va a parar.

Se indicaba más arriba que los atributos fundamentales del relieve eran la longitud y la pendiente. Ambos deben atribuirse al sustantivo ladera, "the most basic landforms" según CLARK y SMALL, (1982), o parafraseando a YOUNG (1972) las formas que constituyen la mayor porción de la superficie de la tierra. Es pues la ladera, superficie inclinada de más o menos pendiente, el elemento de estudio, y dentro de ella la divisoria o punto de inicio de la misma, el objetivo a considerar.

La arroyada en superficie se inicia a partir de un punto desde la divisoria según la teoría de HORTON. Aunque bajo este supuesto existiría una zona sin erosión muy cuestionada (negada por YAIR 1973), no deja de ser un hecho clave considerar la divisoria como un espacio fundamental en la aparición del flujo en superficie.

Frente a la ausencia de flujos continuos en el tiempo, la arroyada cuando existe, hace evolucionar las laderas originando un posible desfase entre unas partes y otras de la misma (la acumulación basal no es exportada). Por esta razón se hace más evidente y apropiado el análisis de la divisoria en aquellos casos en que los cauces no aparecen claramente reflejados.

En el presente trabajo se plantea un análisis topográfico en esta línea de pensamiento, basado en el estudio de las divisorias, en vez de en el análisis de los cauces, como indicadores de las condiciones de génesis de flujo. El trabajo contempla el 
desarrollo de aspectos metodológicos, y se efectúa sobre áreas con materiales contrastados.

Es de advertir finalmente que los resultados no debieran de diferir, al menos en términos relativos, de los que hubieran surgido, en caso de poderse efectuar, mediante el análisis clásico de las redes de drenaje.

Teniendo en cuenta que el agua es el agente fundamental en los procesos analizados, las principales características que definen su papel en la preparación, arrastre y disposición de materiales derivan de las circunstancias siguientes:

- el relieve dirige la dirección del flujo en superficie,

- constituye la superficie por donde éste fluye.

Por tanto, en virtud de ambos aspectos, el relieve es capaz de generar una capacidad de movilización de agua, en unas direcciones, a lo largo de una superficie.

\section{AREA DE ESTUDIO.}

El trabajo se ha realizado en las márgenes de la Depresión de Violada (Huesca), sobre materiales de terrazas fluviales y alternante litología de yesos y margas.

La zona se inscribe morfoclimáticamente dentro del sector semiárido aragonés (RODRIGUEZ VIDAL, 1982) con $500 \mathrm{~mm}$ de precipitación media anual, en la que los procesos de modelado actual están dominados por la presencia de flujos de carácter no permanente.

Topográficamente las dos áreas de trabajo se constituyen en una sucesión de interfluvios y valles de fondo plano en los que no se advierte la excavación de cauces (mapa $n^{2} 1$ ).

Una descripción más detallada de la zona y su historia geomorfológica se puede encontrar en PELLICER y GONZALEZ HIDALGO (1987) y GONZALEZ HIDALGO (1988). 


\section{EL RELIEVE Y LA CAPACIDAD DE MOVILIZACION DEL FLUJO.}

Las divisorias de aguas son los elementos que definen en el espacio los lugares desde donde se origina el flujo; expresan una compartimentación del territorio que significa una capacidad de movilización del agua caída en él. Finalmente constituyen el punto de referencia del área en donde se desarrolla el flujo.

Para su análisis se ha utilizado como documento base los fotogramas aéreos de mayor escala disponible (vuelo 1970, esc. 1:20.000) y como elemento de apoyo la Cartografía Hipsométrica (esc. 1:25.000). Sobre ellos se levantaron los árboles de divisorias en ambos sectores.

El primer resultado fue obtener las unidades de dfrenaje fundamental, vaguadas, $o$ cuencas hidrográficas (Mapa $\mathrm{n}^{\mathrm{Q}} 2$ ).

El paso siguiente ha consistido en la realización de un estudio morfométrico de la configuración de cada una de ellas mediante el estudio de las divisorias. Para ello, se consideraron tres variables:

- Longitud total de las divisorias periféricas a cada vaguada.

- Longitud total de las divisorias internas a las mismas.

- Superficie planimétrica de cada una de ellas.

A continuación se examinaron las siguientes relaciones:

- Longitud Total Divisorias/Area.

- Longitud de Divisorias Interiores/Area.

- Divisorias Periféricas/Divisorias Internas. 


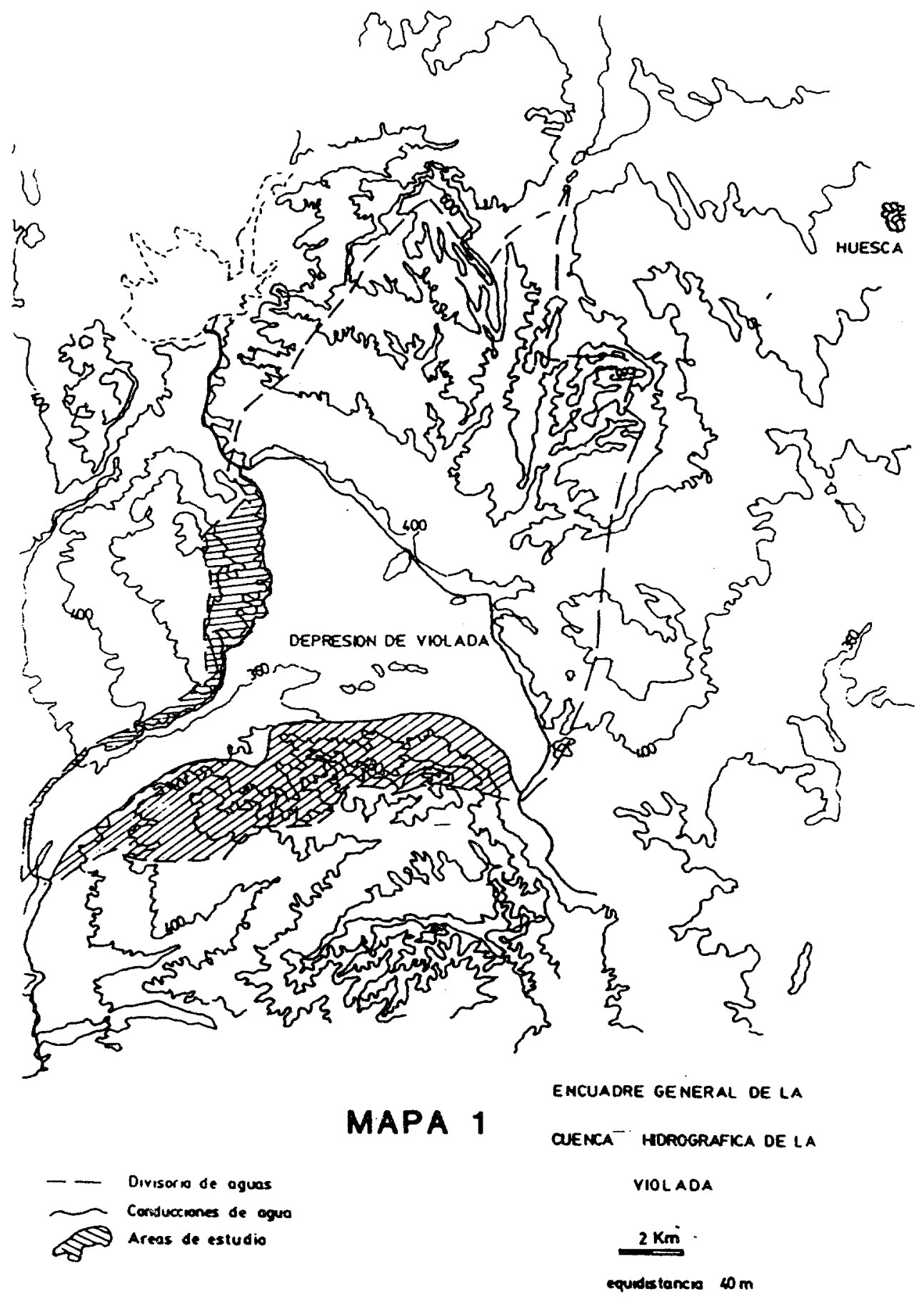




\section{RESULTADOS Y DISCUSION.}

\section{A.- El vigor del relieve.}

La relación Longitud de DIvisorias/Areas, expresa la capacidad total de movilización del agua caída en cada unidad de drenaje y ha recibido el nombre de Vigor de Relieve. En realidad supone una densidad del elemento divisoria.

El cálculo se ha efectuado teniendo en cuenta que las divisorias periféricas afectan solamente en cada unidad de drenaje fundamental a lo largo de una de sus dos laderas, por lo que la ecuación utilizada ha sido:

(Divisorias Periféricas $/ 2)+$ Divisorias Internas

Los resultados se expresan en la Tabla $\mathrm{n}^{2} 1$, col. 6 .

Estos resultados aparecen en la figura 1 en relación a la superficie de cada vaguada, y se puede observar cómo existe una clara homogeneidad en todo el tramo occidental del sector sur, lo que sugiere una posible causa común.

La superposición con la cartografía litológica ha permitido observar como todas estas vaguadas se localizan sobre materiales de terraza. En ellas, la distribución analizada parece respoder a una relación de carácter inverso al área, expresada por una correlación de $-0^{\prime} 89$, a lo largo de una suave pendiente de $-0^{\prime} 33$ (fig., 1, vaguadas 9 a 13).

Por lo que respecta al resto del conjunto, la distribución de los interfluvios en las vaguadas sobre yesos y margas, parece responder además del tamaño a otros criterios.

En el sector oeste las vaguadas más septentrionales toman los valores más altos frente al tramo central y meridional, en los que se aprecia un paulatino descenso de los mismos. 
Por su parte en el sector sur se diferencian claramente un cloque de vaguadas con valores muy altos en todo el tramo central. Desde aquí se da paso a un seftor oriental caracterizado por las disoparidades de este índice.

\section{B.- El encajamiento.}

La relación de ambos tipos de divisorias (Externas e Internas) se ha denominado Indice de Encajamiento. Este índice expresa el modo en que se efectúa la capacidad de movilización total. Es decir, pondera la intervanción de cada tipo de divisorias analizado.

Los valores de esta relación vienen dados de la siguiente manera:

$$
\begin{aligned}
& \text { I.E. }>1=\text { D.P. }>\text { D.I. } \\
& \text { I.E. }=1=\text { D.P. }=\text { D.I. } \\
& \text { I.E. }<1=\text { D.P. }<\text { D.I. }
\end{aligned}
$$

Siendo:

I.E. Indice de Encajamiento

D.P. Longitud de las Divisorias Periféricas, (km lineales)

D.I. Longitud de las Divisorias Inferiores, (id.)

Su significado estriba en el hecho de que si los valores son elevados, la movilización de agua en superifice tendrá una tendencia a la incisión de carácter lineal por la escasa compartimentación existente. Por el contrario, grados de encajamiento bajos significarán una posible pérdida de energía de los aportes de agua, aunque exista una capacidad superior de movilización total por un aumento de divisorias internas.

Se plantea además la hipótesis de cómo podrá variar la respuesta hidrológica en virtud del tipo de precipitación. A mayores cantidades de lluvia serían las áreas de menor índice las que estarían en condiciones de generar un movimiento más generalizado, ya que su mayor compartimentación interna sería capaz de generar una mayor energía.

Mientras tanto, con aportes menores ocurriría a la inversa. Serfan las vaguadas de valores altos de encajamiento las que estarán en mejores condiciones de movilizar el agua, pues no existirá una dispersión del flujo por presentar una menor cormpartimentación. El resultado sería que podría generarse una mayor concentración de energía. 
Por consiguiente se plantea la hipótesis de las posibles diferencias en las respuestas temporales de cada tipo de vaguada acorde a los tipos de precipitaciones, y por tanto muy probablemente variables a lo largo del tiempo.

Las gráficas de distribución del mencionado índice con respecto a las áreas permiten nuevamente establecer diferentes comportamientos según se trate de vaguadas sobre yesos -margas o terrazas-.

Si bien en el caso de las vaguadas en terraza la distribución parece estar acorde al tamaño, (correlación -0'90, Fig. 2), en el caso de las vaguadas sobre el segundo tipo de materiales la distribución de los valores de encajamiento parece sugerir dos tipos de comportamiento distinto. Por un lado las vaguadas que se localizan a la izquierda de la gráfica, las más pequeñas, se distribuyen a lo largo de una pendiente muy acusada, mientras las restantes adoptan una distribución más laxa, aunque también mantienen la pauta general de unos mayores valores con las menores áreas.

Se advierte la tendencia a un mayor encajameinto en las de menor tamaño, de tal modo que pudiera intuirse dos tipos de distribución (en este caso si en función del área). Quizá pudiera ser interpretado como diferentes estadios de evolución en función de la proximidad a los niveles de base respectivos, independientemente de las diferencias hipsométricas que pudieran existir respecto a ésta.

En este sentido es de notar como vaguadas con áreas similares y diferencias hipsométricas distintas (10-12 m en vag. $n^{2} 25,26 ; 60-70 m$ en vag. $n^{2} 14,16$ etc.), reflejan valores muy parecidos en los Indices de Encajamiento.

\section{C.- La compartimentación del relieve.}

En la relación Divisorias Internas/Area, se ha buscado matizar la posible influencia que tuviese la longitud de las divisorias periféricas, al haberse comprobado la falta de relación con las superficies definidas por las mismas.

De este modo se ha buscado analizar el comportamiento interno de cada vaguada en lo que se ha denominado Compartimentación de relieve, nuevamente una expresión de densidad de divisorias. 
En la Fig. 3 se refleja el conjunto de las vaguadas analizadas, y se observa la existencia de dos comportamientos distintos.

Las vaguadas en terrazas presentan similares grados de compartimentación interna (medidos en densidad de divisorias) en relación al área. La ausencia de correlación con el tamaño se interpreta como resultado de una escasa jerarquización de los flujos en superficie, atribuida a la elevada infiltración de estos materiales.

Mientras tanto las vaguadas sobre materiales margosos y yesíferos ofrecen unos valores superiores a los anteriores, volviéndose a notar una ausencia de relación clara con el área.Al contrastar los km lineales con el área de cada vaguada, (Fig. 4), los diferentes grados de incisión se pueden expresar mediante las diferentes pendientes de ambas agrupaciones (ambas con elevadas correlsaciones: yesos y amrgas 0'96; terrazas 0'99). En yesos y margas la pendiente es de 10'36, mientras en terrazas es solamente de 4'4. Los diferentes interceptos de ambos conjuntos se interpretan como las áreas mínimas, a partir de las cuales la articulación interna del flujo superficial comienza a realizarse.

Por tanto es fácil deducir para cada uno de ellos un comportamiento, atribuido en su mayor parte a las diferentes condiciones de infiltración (superiores en las terrazas).

La consecuencia que es extrae de este hecho es la cuantificación morfométrica del diferente grado de incisión que se realiza en los dos materiales analizados. De ello es buena prueba que con similares áreas las vaguadas excavadas en yesos presxenten un grado de compartimentación superior a sus homónimas en terraza. Y como consecuencia, la mejor disposición a la génesis del flujo en superficie sobre los materiales margoyesíferos en la alternancia de yesos y margas que en los sectores sobre terrazas.

\section{CONCLUSIONES}

La recapitulación a que conduce el análisis previo lleva a considerar que las mediciones efectuadas sobre las divisorias de aguas permiten diferenciar áreas con distintas capacidades de movilización de flujo, así como plantear la hipótesis de diferentes tipos de respuesta según las magnitudes pluviométricas. 
A su vez, dado que en realidad no se puede hablar de una red de drenaje típica por estar localizada en un área semiárida-árida, los datos manejados expresan mejor las condiciones del desarrollo del flujo que si se hubieran tratado al modo tradicional (análisis de la red de drenaje canalizado).

Los resultados difieren según el tipo de material litológico, extensión y situación espacial.

De los tres indicadores manejados, el índice de encajamiento, o relación entre divisorias periféricas y divisorias internas, parece ser la mejor relación para describir el diferente comportamiento de litologías distintas ante los procesos hídricos superficiales.

\begin{tabular}{|c|c|c|c|c|c|c|c|}
\hline & TABLA & 1 & MORFOMETR & IIA DE L & AS VAGU & As. & \\
\hline 1 . & 2. & 3. & 4. & 5. & 6. & 7. & 8. \\
\hline $\begin{array}{l}01- \\
02- \\
03- \\
04- \\
05- \\
06- \\
07- \\
08- \\
09= \\
10= \\
11= \\
12= \\
13= \\
14- \\
15- \\
16- \\
17- \\
18- \\
19- \\
20- \\
21- \\
22- \\
23- \\
24- \\
25- \\
26-\end{array}$ & $\begin{array}{l}1.200 \\
0.875 \\
0.900 \\
1.434 \\
3.250 \\
1.425 \\
1.750 \\
2.924 \\
5.550 \\
1.500 \\
4.350 \\
4.000 \\
1.500 \\
1.430 \\
1.350 \\
0.900 \\
0.900 \\
5.300 \\
2.600 \\
6.000 \\
4.250 \\
1.625 \\
4.125 \\
1.500 \\
1.250 \\
0.625\end{array}$ & $\begin{array}{r}2.8 \\
1.0 \\
1.0 \\
6.0 \\
11.7 \\
6.0 \\
2.5 \\
12.3 \\
22.7 \\
2.1 \\
13.3 \\
11.3 \\
2.7 \\
5.3 \\
2.4 \\
3.2 \\
1.0 \\
10.6 \\
4.6 \\
17.2 \\
20.4 \\
9.2 \\
31.0 \\
3.0 \\
3.7 \\
1.2\end{array}$ & $\begin{array}{l}0.514 \\
0.260 \\
0.095 \\
0.654 \\
1.396 \\
0.700 \\
0.470 \\
1.703 \\
5.079 \\
0.536 \\
3.091 \\
2.278 \\
0.583 \\
0.703 \\
0.420 \\
0.276 \\
0.136 \\
1.187 \\
0.498 \\
1.876 \\
1.775 \\
0.800 \\
2.870 \\
0.860 \\
0.383 \\
0.148\end{array}$ & $\begin{array}{r}4.00 \\
1.875 \\
1.900 \\
8.134 \\
14.950 \\
7.925 \\
4.250 \\
15.224 \\
28.250 \\
3.600 \\
17.650 \\
15.300 \\
4.200 \\
6.730 \\
3.750 \\
4.100 \\
1.900 \\
15.900 \\
7.200 \\
23.250 \\
24.700 \\
10.875 \\
35.125 \\
4.500 \\
5.000 \\
1.875\end{array}$ & $\begin{array}{r}7.78 \\
7.21 \\
20.00 \\
12.43 \\
6.95 \\
11.32 \\
9.04 \\
8.93 \\
5.56 \\
6.71 \\
5.71 \\
6.71 \\
7.20 \\
9.57 \\
8.92 \\
14.85 \\
13.97 \\
13.39 \\
14.45 \\
12.39 \\
13.91 \\
13.59 \\
12.23 \\
5.23 \\
13.05 \\
12.86\end{array}$ & $\begin{array}{l}0.42 \\
0.85 \\
0.90 \\
0.33 \\
0.27 \\
0.30 \\
0.70 \\
0.20 \\
0.24 \\
0.70 \\
0.32 \\
0.35 \\
0.55 \\
0.27 \\
0.58 \\
0.28 \\
0.90 \\
0.50 \\
0.56 \\
0.34 \\
0.20 \\
0.17 \\
0.13 \\
0.50 \\
0.33 \\
0.50\end{array}$ & $\begin{array}{r}5.44 \\
3.83 \\
10.52 \\
9.17 \\
8.38 \\
8.57 \\
5.31 \\
7.22 \\
4.46 \\
3.91 \\
4.30 \\
4.96 \\
4.63 \\
7.53 \\
5.71 \\
11.59 \\
7.35 \\
8.93 \\
9.23 \\
9.16 \\
11.49 \\
11.50 \\
10.80 \\
3.48 \\
9.66 \\
8.10\end{array}$ \\
\hline
\end{tabular}

1. Identificación de las vaguadas, $(=)$ Terrazas, $(-)$ Margas/Yesos; 2. (Longitud del perlmetro $/ 2$ ); 3 . Divisorias internas, $\mathrm{Km}$; 4 . Area Km?; 5 . $\mathrm{Km}$ totales $(2+3) ; 6$. Vigor de Relieve, $(5 / 4) ; 7$. Encajamiento, $(2 / 3) ; 8$. Compartimentación, (3/4). (en G HIDALGo $1988)$. 
Figura 1.

Vigor de Relieve

Vaguadas en Yeso/Margas y Terrazas

- necosimeroes 0 Terrazes

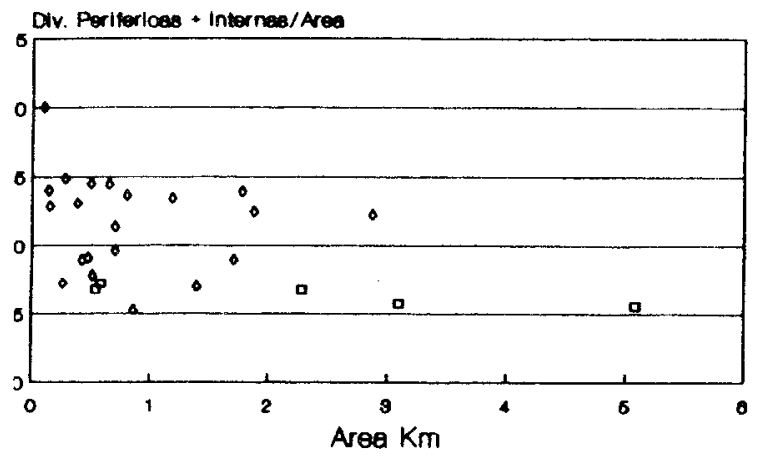

Figura 3.

Compertimentacion

Vaguadas en Yeso/Margas y Terrazas

- vecos/meroes o Torreans

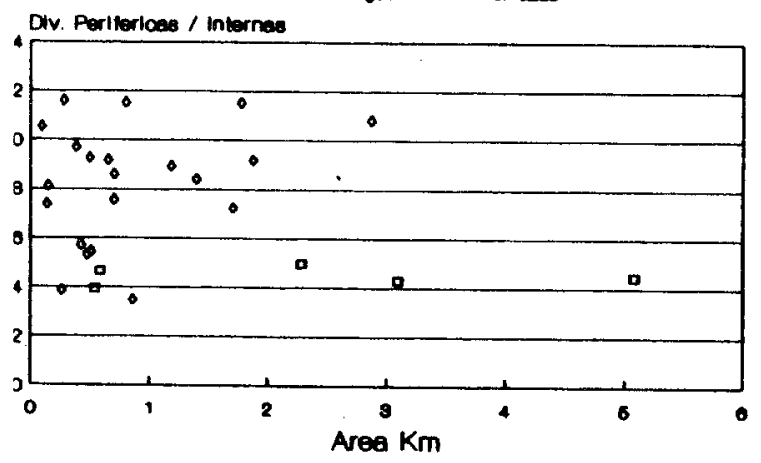

Figura 2.

Encajamiento

Vaguadas on Yeso/Margas y Terrazas

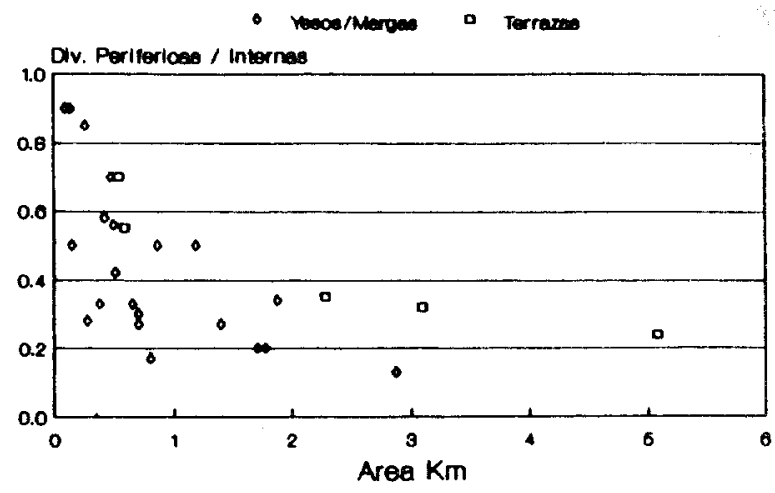

Figura 4

Divisorias en $\mathrm{Km}$ lineales

Totales e Internas

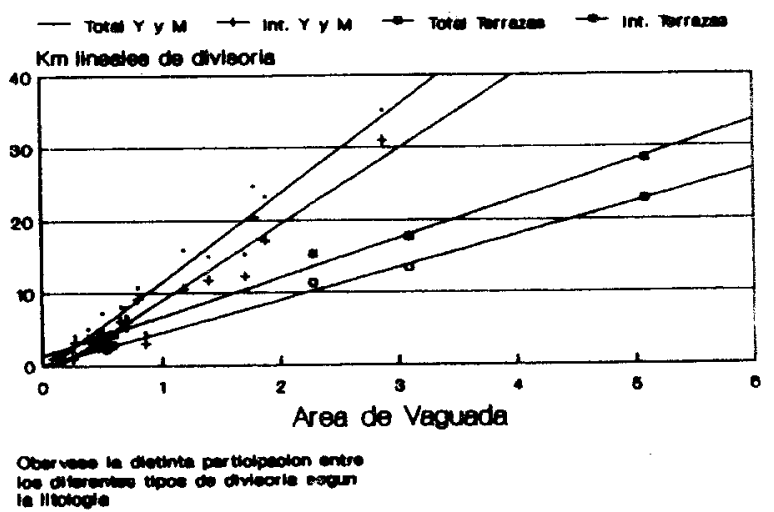




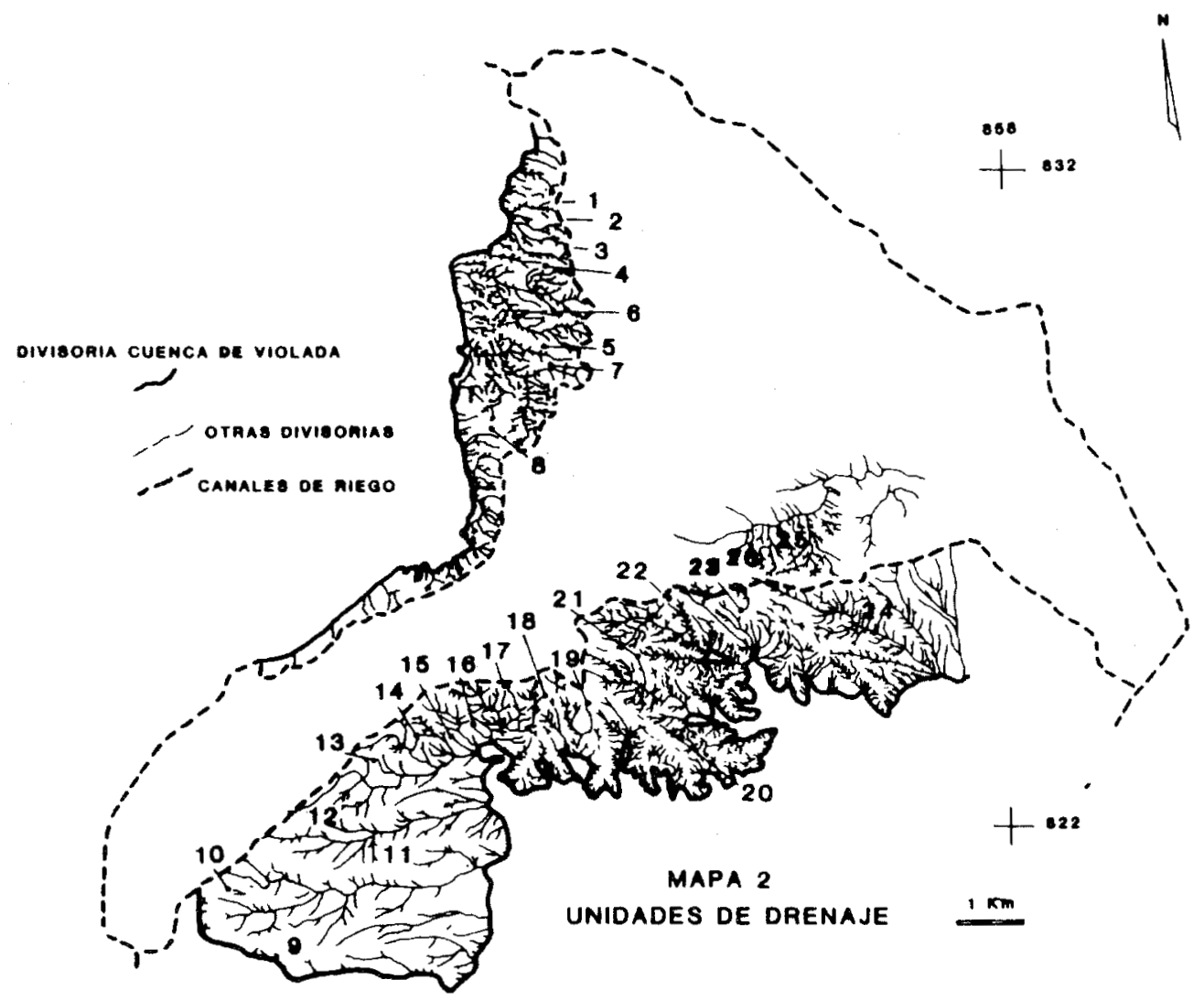




\section{BIBLIOGR AFIA.}

AVENA, G.C., GIULIANO, G., y PALMIERI, E.L. (1967): Sulla valutazione quantitativa della gerarchizzazione ed evoluzione del reticoli fluviali. Boll. Soc. Geol. Italiana, 86, 781-796.

BAND, L. (1985): Field parametrization of an empirical sheetwash transport equation. Catena, 12, 281290.

CLARK, M. y SMALL, J. (1982): Slopes and Weathering. Cambridge Univ. Press, 112 p.

GONZALEZ HIDALGO, J.C. (1988): Geomorfología y procesos morfogenéticos en las márgenes del Polígono de Riegos de la Violada. Aproximación metodológica. Tesis Msc. IAMZ, $239 \mathrm{p}$. Zaragoza.

GONZALEZ HIDALGO, J.C. (1989): Introducción al estudio del drenaje en superficie de las Sierras Exteriores Oscenses. Sector Isuela-Vero. Rev. Lucas Mallada. 1, 67-80.

GOUDIE, A., ANDERSON, M., BORT, T., LEWIN, J., RICHARDAS, K., WHALLEY, B. Y WOESLEY, B.W. (1981): Geomorphological techniques. Allen and Unwin, 395 p. London.

GREGORY, K.J. (1976): Drainage networks and climate. In DERBYSHIRE, E., (Ed.) Geomorphology and climate. John Willey, 289-318.

JARDI, M. (1986): Cálculo del valor relativo de la erosión en cuencas de pequeño tamaño. Cuadernos de Inv. Geogr., XIII, (1-2), 3-10.

JONES, J.A. (1987): The initiation of natural drainage network. Prog. in Phys. Geogr., 11, (2), $207-$ 245.

KNGHTON, D. (1984): Fluvial forms and processes. Ed. Arnold, 218 p. London.

MITCHELL, J.K. y BUBENZER, G.D. (1980): Soil loss estimation. In KIRKBY, M.J. and MORGAN, R.P.C., (Eds.) Soil Erosion. Willey and Son, 18-62.

MOPU (1982): Guía para la elaboración de estudios del medio fisico. C.E.O.T.M.A. Manuales No 3, 572 p. Madrid.

MORGAN, R.P.C. (1976): The role of climate in the denudation system: a case study of West Malaysia. In DERBYSHIRE, E. (Ed.) Geomorphology and Climate. John Willey, 319-344.

MORGAN, R.P.C. (1979): Soil erosion and conservation. Ed. Longman, 117 p.

MORGAN, R.P.C. (1980): Implications. In KIRKBY, M.J. and MORGAN, R.P.C. (Eds.) Soil Erosion. Willey and son, 252-292.

PELLICER, F. y GONZALEZ HIDALGO, J.C. (1987): Depósitos del Somontano Oscense en la Depresión de la Violada. Actas $X$ Congreso AGE, Dpto. Geografía Univ. de Zaragoza, T. I, 47. 56.

RODRIGUEZ VIDAL, J. (1982): Distribución morfoclimática en la depresión media del Ebro: procesos dominantes y modelado actual. Est. Geol. (38), 43-50. 
THORNES, J.B. (1976): Semiarid erosional systems: case studies from Spain. L.S. Econ. and Pol. Sc. Geographical Paper $n^{2} 7,79 \mathrm{p}$.

THORNES, J.B. y GILMAN, A. (1983): Potential and actual erosion around archeological sites in south east Spain. Rainfall simulation, runoff and soil erosion. Catena suppl. , 4, 91-112.

WISCHMEIER, W.H. y SMITH, D.D. (1960): A universal soil loss equation to guide conservation farm planning. Trans. 7th Inter. Congr. of soil Science, 1, 418-425.

YAIR, A. (1973): Theoretical considerations on the evolution of convex hillslopes. Z. für Geomorph. Supp. Bnd., 18, (1-9), 177-189.

YOUNG, A. (1972): Slopes. Ed. Oliver and Boyd, 288 p. Edinburgh. 